\title{
Endocrine-like cells in the terminal bronchioles and saccules of human fetal lung: an ultrastructural study
}

\author{
JENNIFER McDOUGALL
}

From the Department of Morbid Anatomy, University College Hospital Medical School, London WC1

\section{McDougall, Jennifer (1978). Thorax, 33, 43-53. Endocrine-like cells in the terminal} bronchioles and saccules of human fetal lung: an ultrastructural study. An ultrastructural study of Kulschitsky cells was made on lung tissue from five human fetuses of gestational ages between 18 and 25 weeks. The cells were found to occur with relative frequency throughout the bronchi and bronchioles, as well as in the developing saccules. This report describes in detail the cells of the terminal bronchioles and saccules, where their long cytoplasmic processes weave through the epithelium and come into contact with intercellular spaces and the lumen. The cytoplasmic organelles have an orderly arrangement comparable to secreting cells. Dense core vesicles characterise the cytoplasm but clear vesicles and some with less dense, diffuse cores are also present, and may be seen outside the plasma membrane and in the interstitial spaces. The K-cells are usually closely associated with capillaries or smooth muscle cells across the basement membrane. In addition, the rare occurrence of a small unmyelinated nerve fibre in the vicinity of a K-cell in a terminal bronchiole raises the possibility that the cells fulfil a type of 'mini receptor' role in addition to their probable vasoactive one. Evidence is given that $\mathrm{K}$-cells divide in human fetal lung.

Rare cells of 'unusual morphology located within the epithelium' were described by Campiche et al. (1963) in their electron microscopic study of human fetal lung. The ultrastructure of these cells, designated as $\mathrm{K}$-cells (Kulschitsky cells) or endocrine-like cells, has been further studied by Cutz and Conen (1972), Hage (1972a, b) and Rosan and Lauweryns (1972). These studies of human fetal and adult lung (Terzakis et al., 1972), together with those on animals (Hung et al., 1973; Lauweryns et al., 1973; Cutz et al., 1974; Jeffery and Reid, 1975) have been confined to the conducting epithelium of the lung. Lauweryns (1970) has mentioned the presence of neurosecretory cells among 'respiratory epithelia' in premature infants.

The observations reported here were made on five human fetuses in the gestational age range 18 to 25 weeks. The differentiation of type 1 (T1) and 2 (T2) pneumocytes in this material has been reported elsewhere (McDougall and Smith, 1975). In that study, the presence of K-cells was not recorded. The present paper describes their presence not only in the proximal airways but also in the terminal bronchioles and saccules, where their cytoplasmic processes apically make contact with the lumen and basally are adjacent to developing vessels. The functional significance of these findings is discussed.

\section{Material and methods}

Lung samples were obtained from five human fetuses of 18-25 weeks' gestational age after abortion by hysterotomy. Portions of lung, $1 \mathrm{~mm}^{3}$ in size, from representative peripheral and hilar regions were taken for electron microscopy. They were fixed overnight in $4 \%$ glutaraldehyde in $0.125 \mathrm{M}$ phosphate buffer, $\mathrm{pH} 7.2$ at $4^{\circ} \mathrm{C}$, washed, and stored in fresh $0.125 \mathrm{M}$ phosphate buffer at $4^{\circ} \mathrm{C}$. The tissue was post-fixed in $1 \%$ osmium tetroxide in $0.125 \mathrm{M}$ phosphate buffer for two hours, washed, dehydrated, and embedded in Epon. Sections of $0.5-1.0 \mu \mathrm{m}$ were stained with toluidine blue; ultrathin sections were stained with uranyl acetate and lead citrate and examined in a Philips 200 electron microscope.

\section{NOMENCLATURE}

Kulschitsky cell: synonyms used are K-cell, endocrine-like cell, Feyrter cell.

Airways: those passages lined by conducting epithelium. 
Terminal bronchioles: in the fetus those bronchioles lined by cuboidal epithelium from which the saccules develop and into which capillaries are incorporated.

Saccules: the prospective respiratory areas of the lung lined by $\mathrm{T} 1$ and $\mathrm{T} 2$ pneumocytes.

Pseudoglandular period: that period of gestation (5-17 weeks) when the bronchial tree is laid down.

\section{Results}

The frequent occurrence of $\mathrm{K}$-type cells in the fetal lungs is striking. With the exception of the primitive buds and ducts of the pseudoglandular period, they are seen either singly or in groups of two or more over the whole of the age range studied in all developing bronchi, bronchioles, and saccules.

The basic K-cell structure consists of a round to elliptical nucleus, with patchy dense chromatin and well-differentiated cytoplasm filled with organelles (Fig. 1). At any of the gestational ages studied, the structure appears more electron-dense than that of neighbouring epithelial cells. The characteristic feature is a large number of dense core vesicles of diameter 75-200 $\mu \mathrm{m}$. Typically, the dense core is surrounded by an electronlucent halo. However, there is great variation in density of the cores and some are diffuse and have no apparent halo (Fig. 2). In addition, most cells have several clear vesicles of similar diameter without any dense core. Much of the electron density of the cytoplasm may be attributed to the presence of numerous polysomes and free ribosomes. Small round or elliptical mitochondria are common but their numbers seem to vary inversely with those of the dense core vesicles. This may simply reflect the polarity of organelles within the cell (described below) and may be emphasised by sectioning. Most cells have a well-developed Golgi complex with numerous small vesicles. Cisternae of rough endoplasmic reticulum are short and inconspicuous. Dense bodies, singly or in groups, are seen infrequently, and lipid droplets are rare. By comparison with neighbouring undifferentiated epithelial cells, glycogen particles within the K-cell cytoplasm are also rare, but they do occur mainly in the $\alpha$ form. Some cells may have bundles of short microfilaments, usually around the nucleus.

The arrangement of the organelles in the $\mathrm{K}$ cells is orderly and probably indicative of the order of synthesis of the vesicles in a secreting cell (Fig. 3). There is an extensive perinuclear zone of Golgi vesicles, smooth endoplasmic reticulum, and, sometimes, bundles of microfilaments. These are surrounded by the mitochondria and some cisternae of rough endoplasmic reticulum. The dense core vesicles and some clear vesicles occur at the periphery and may be more numerous at the base of the cells.

Contact between the endocrine-like and other के epithelial cells of the airways and saccules is $\overrightarrow{0}$ similar to that between epithelial cells. There is lateral plasma membrane infolding with the formation of interdigitating microvilli. The junctions formed are usually desmosomes, but where occasionally the cytoplasm reaches or comes near to the lumen, tight junctional complexes are formed (Fig. 4). Within the K-cells pinocytotic vesicles are frequently seen, some opening as flask-like pits into the plasma membrane. There is some variation in the structure of $\mathrm{K}$-cells in different parts of the respiratory apparatus. Generally in the conducting passages the shape of the cells is more oval and there appears to be less lateral plasma membrane interdigitation with the neighbouring columnar epithelial cells. The organelles show more definite arrangement within the cytoplasm. Whorls of endoplasmic reticular membrane usually without attached ribosomes (Fig. 5) may be seen, but not in the more distal cells. Although cells may occur singly, more often they are seen in small groups.

The significant observations in this study are those of cells occurring in the prospective respiratory portion of the lung-the terminal bronchioles and saccules. They usually appear as single cells with long cytoplasmic processes which wander away from their cell bodies. These processes can be identified between other epithelial cells, apparently projecting into them, and in the intercellular spaces (Figs 1 and 6a). These processes usually have many more dense core vesicles than the cytoplasm of the cell body, indicating the polarity of organelles within the cytoplasm.

The cell bodies are usually triangular or round $\frac{D}{O}$ in shape and have cytoplasmic organelles, although bundles of microfilaments are rarely seen. There $N$ is usually some interdigitation of lateral plasma membranes.

The cells lie on the epithelial basement mem- $\omega$ brane and extend towards the lumen, occasionally coming into contact with it. Only a small portion of the cytoplasm is usually exposed as a tuft of $\mathbb{D}$ microvilli. The remainder is 'protected' by processes from cuboidal cells or T1 and T2 pneumocytes, with which definite tight junctions are $\underset{\mathbb{D}}{\stackrel{D}{ }}$ formed (Figs 4, 6a and 6b). Most often the cells $\frac{?}{\overparen{D}}$ and processes are covered completely at the $\varrho$ luminal end by at least a thin cytoplasmic extension from an epithelial cell. 


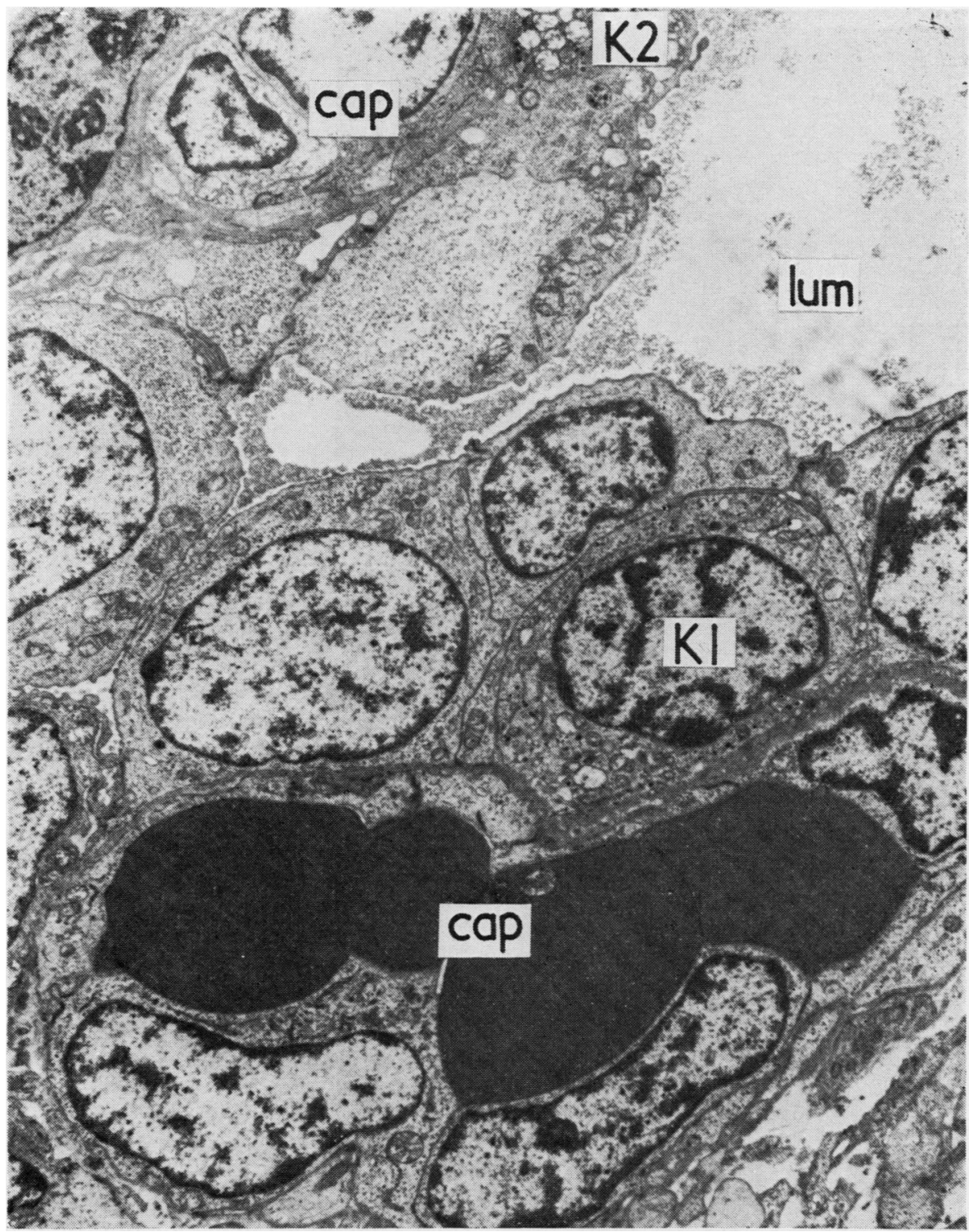

Fig. 1 23-week fetus. Survey of a terminal bronchiole with capillaries (cap) incorporated into its wall and cuboidal cells lining its lumen (lum). $A K$-cell $(K 1)$ and $K$-cell process (K2) can be indentified by their dense core vesicles and mitochondria. They are closely associated with the capillaries. $\times 5200$. 


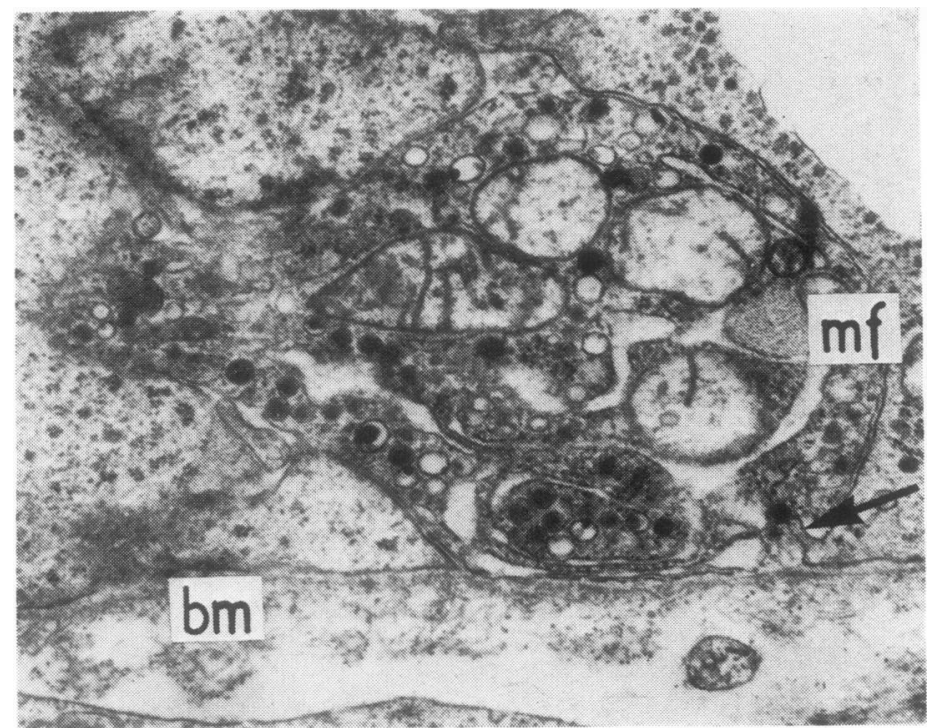

Fig. 2 23-week fetus. A K-cell cytoplasmic process on the basement membrane of a terminal bronchiole. It contains numerous dense core (of varying density) and clear vesicles and a bundle of microfilaments $(\mathrm{m} f)$. Although the epithelial basement membrane (bm) is intact, the basal plasma membrane of the process appears discontinuous, and two granules appear external to it $(\uparrow)$. $V$ esicles can also be seen in the intercellular spaces. $\times 21600$.

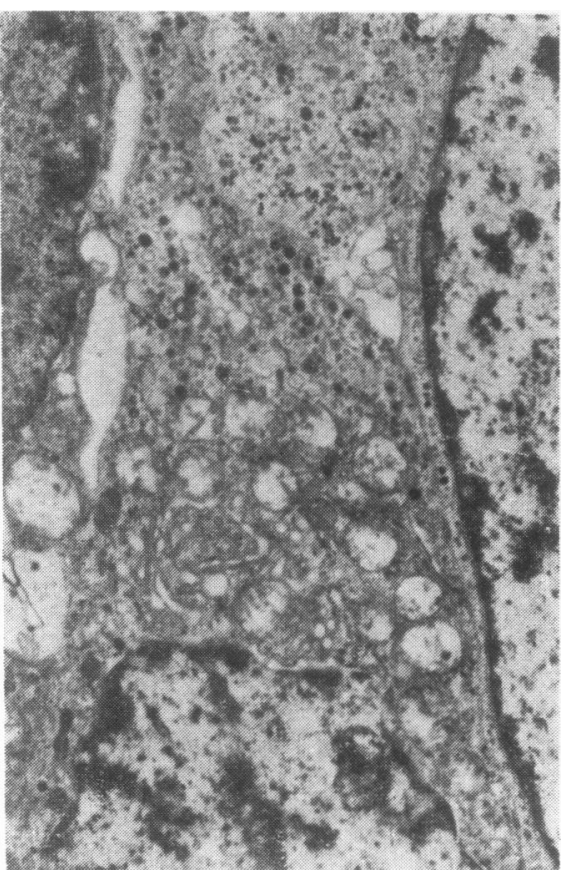

Fig. $3 A$ bronchiolar $K$-cell in a 22-week fetus. The cytoplasmic organelles have a definite arrangementa perinuclear zone of Golgi vesicles and smooth endoplasmic reticulum surrounded by mitochondria. Dense core vesicles and clear vesicles lie in the periphery. $\times 12650$.
In the prospective respiratory area, the cells juxtaposed to the basement membrane of the Kcells on the interstitial side are either endothelial cells of capillaries incorporated into the terminal bronchioles and saccules, or developing smooth muscle cells of the bronchiole walls. As a high proportion of the K-cells and their processes observed were adjacent to these capillaries a detailed study was made of them. Discontinuities were observed in the basal plasma membrane so that the cytoplasm and, particularly, the dense core vesicles appeared to be in direct contact with the basement membrane and interstitium (Fig. 2). Where the plasma membrane appeared intact, dense core and clear vesicles appeared to be fused with it or directly outside it (Fig. 7a). Dense core and clear vesicles were also seen in intercellular spaces (Fig. 7b).

No nerve processes were seen in the saccule walls. However, a small unmyelinated fibre was seen in the wall of a terminal bronchiole lined by cuboidal epithelium in the vicinity of a $\mathrm{K}$-cell (Figs 8 and 9). Examination of several consecutive sections did not reveal any connection with it. As Fig. 8 shows, this particular endocrine-like cell has the typical appearance with extensive Golgi vesicles and lamellae, numerous mitochondria, and many dense core vesicles predominantly in the basal cytoplasm and close to the plasma membrane. A small portion of the apical cytoplasm appears to extend to the lumen and forms tight 


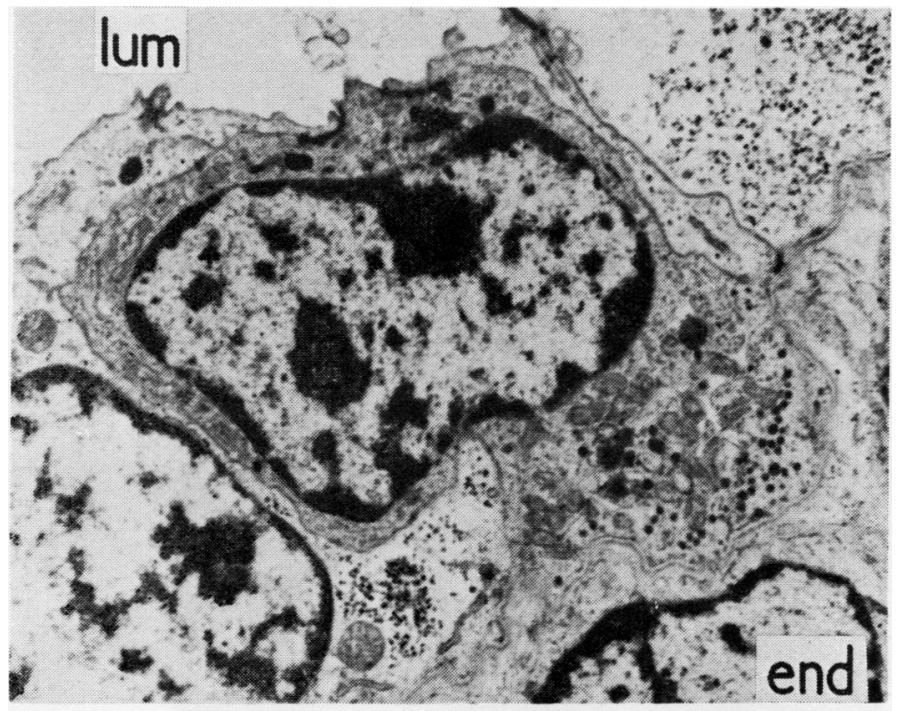

Fig. 4 19-week fetus. A K-cell in a terminal bronchiole. A portion of the apical cytoplasm protrudes at the lumen, and tight junctions connect the cell with adjacent cuboidal cells. Dense core vesicles occur, mainly in the basal cytoplasm, which is associated across the basement membrane with the endothelium (end) of a capillary developing in the bronchiole wall. $\times 12650$.

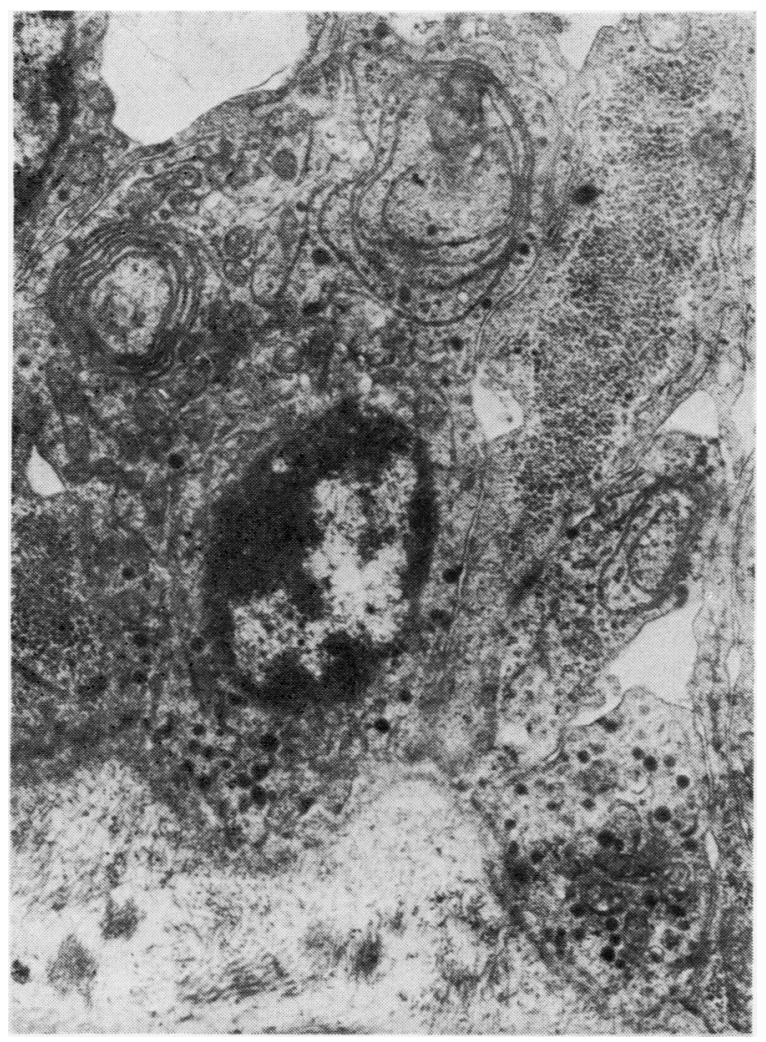

Fig. 5 19-week fetus. A bronchiolar K-cell and process with characteristic dense core vesicles. In addition, there are whorls of ribosome-free membrane within the cytoplasm. $\times 14500$. 


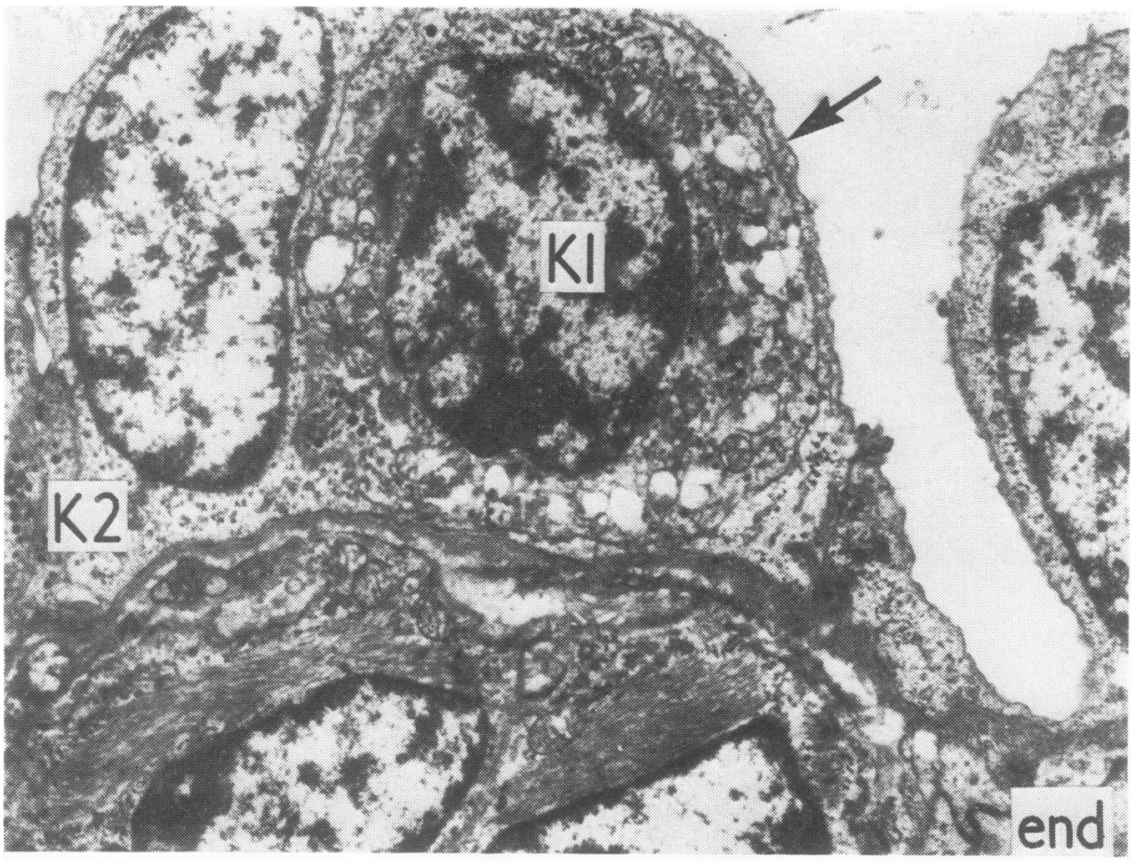

(a)

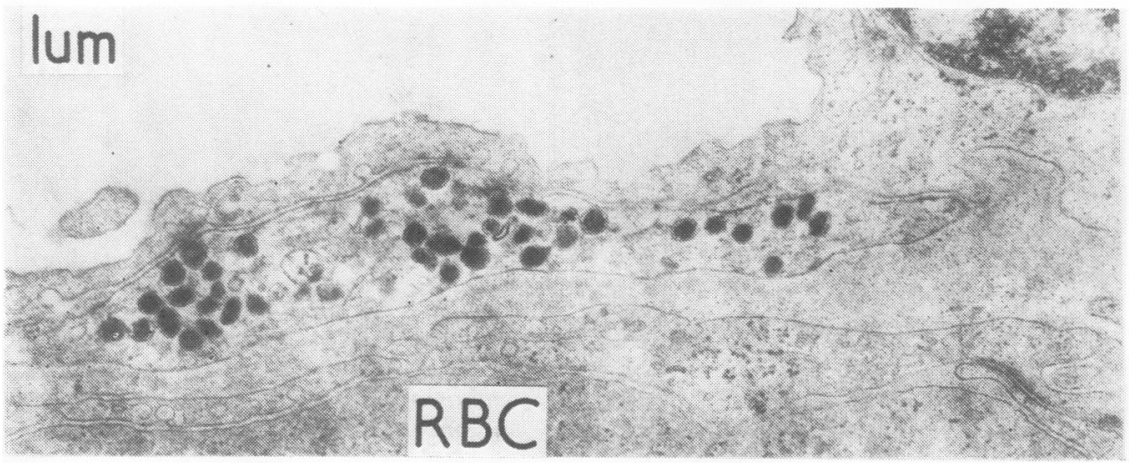

(b)

Fig. 6 (a) 22-week fetus. A K-cell $(K 1)$ and process $(K 2)$ at the junction of a terminal bronchiole and saccule. The process appears between two adjacent cuboidal cells. The cell body is 'protected' from the lumen by a thin cytoplasmic process ( $\uparrow$ ) of a cuboidal epithelial cell. Note the smooth muscle cells in the bronchiolar wall and part of a capillary (end) with epithelial thinning above it. $\times 8400$. (b) 23-week fetus. The attenuated cytoplasm of a T1 pneumocyte overlies a $K$-cell process with numerous dense core vesicles. The basement membrane of the adjacent capillary (with red blood cell $(R B C))$ endothelial cells appears fused with that of the epithelial cells. $\times 27000$. 


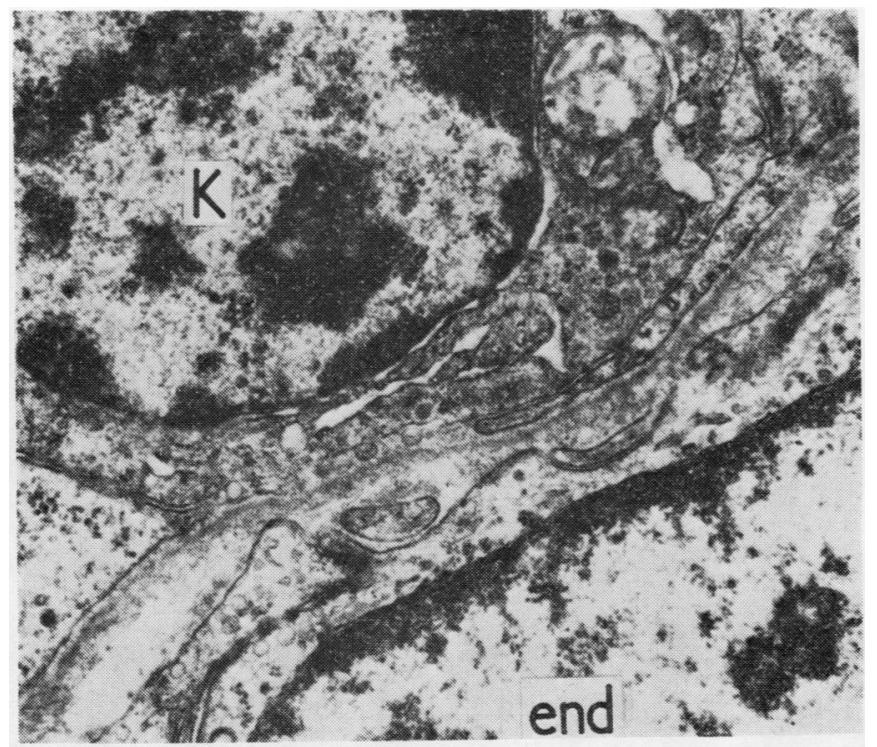

(a)

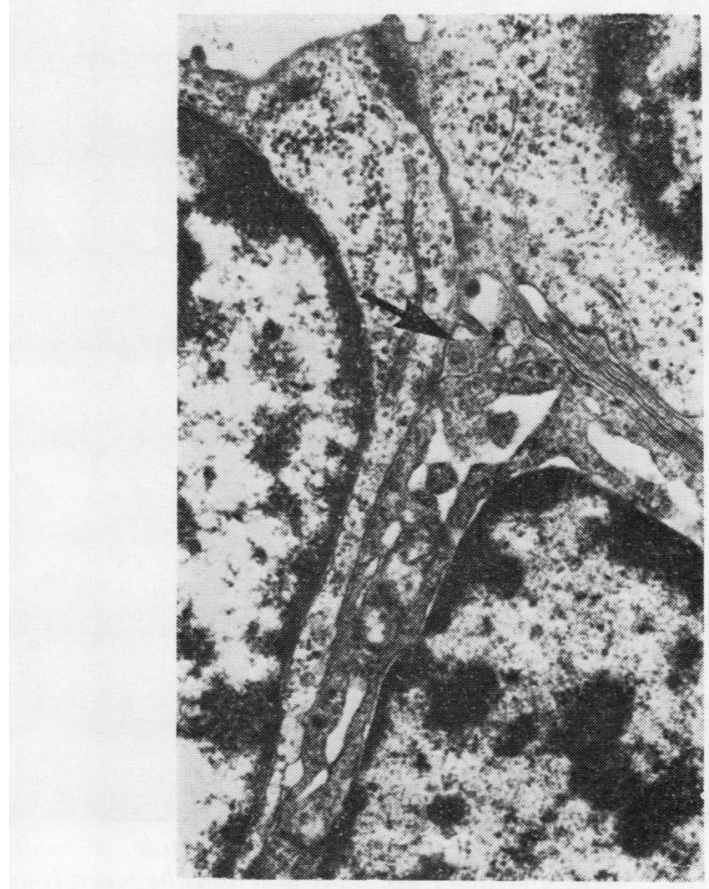

(b)

Fig. 7 (a) 23-week fetus. The base of a K-cell (K) on the epithelial basement membrane adjacent to a capillary. Vesicles are seen external to the cell plasma membrane near the basement membrane. $\times 21600$. (b) 23-week fetus. Dense core vesicles and vesicles $(\uparrow)$ external to the apical plasma membrane of a K-cell in a terminal bronchiole. $\times 17100$. 


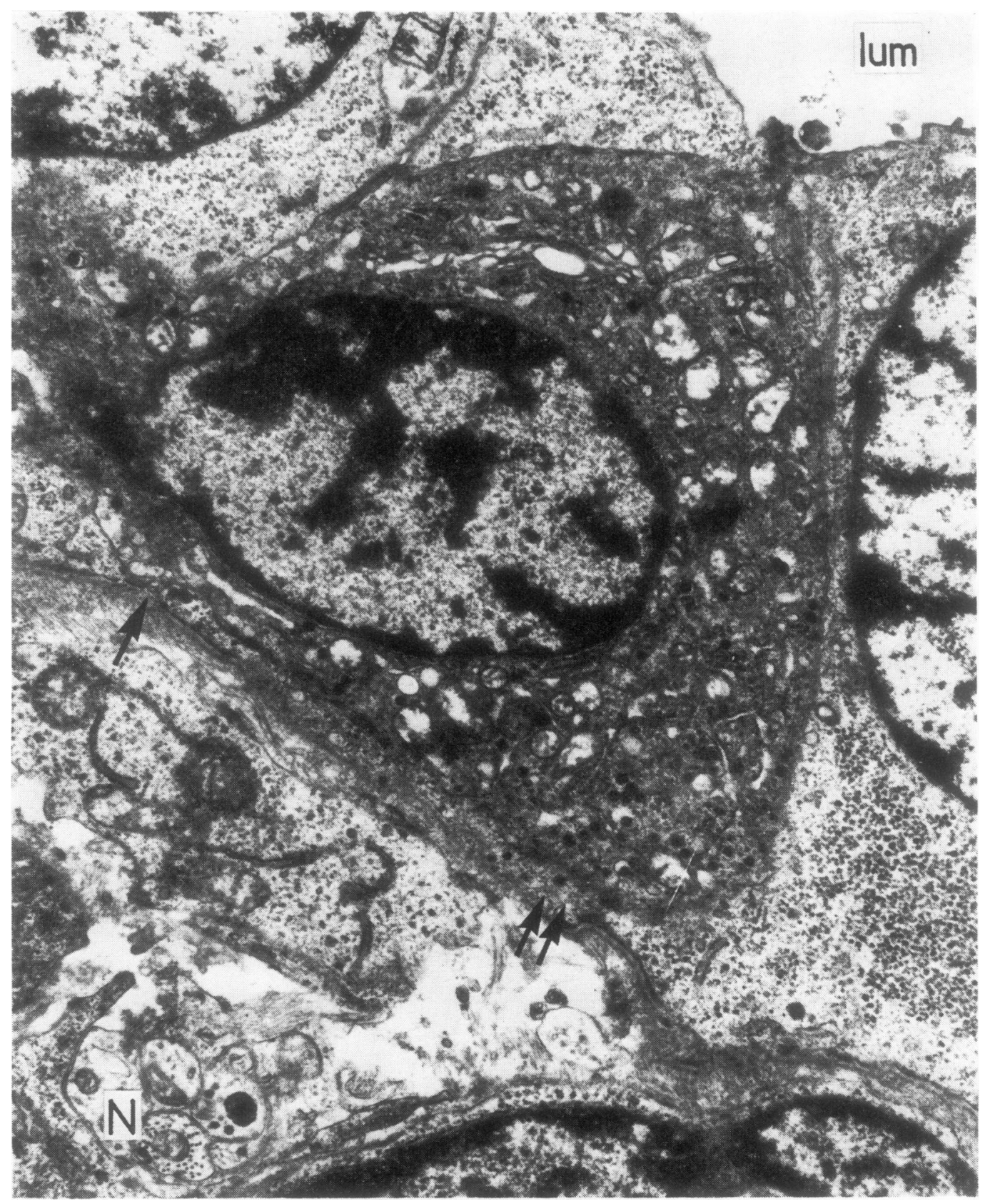

Fig. 8 23-week fetus. A triangular-shaped $K$-cell in a terminal bronchiole. A small portion of the apical cytoplasm is exposed at the lumen, and there are tight junctions between it and adjacent cuboidal epithelial cells, and desmosomes at lateral connections. The cytoplasmic organelles are characteristically arranged with Golgi lamellae and vesicles around the nucleus. Numerous mitochondria and dense core vesicles predominate in the basal cytoplasm. Several dense core and clear vesicles are external to the cell and are either in direct connection with the basement membrane $(\uparrow)$ or apparently passing through the thin extension of cytoplasm of the cuboidal epithelial cell $(\uparrow \uparrow)$. In the interstitium is a small nerve $(N)$ within an arch of cytoplasm of an undifferentiated interstitial cell. Mitochondria and microtubules are seen, and in one process are three dense core vesicles of dimensions corresponding to the lower range of the $K$-cell dense core vesicle. $\times 14000$. 


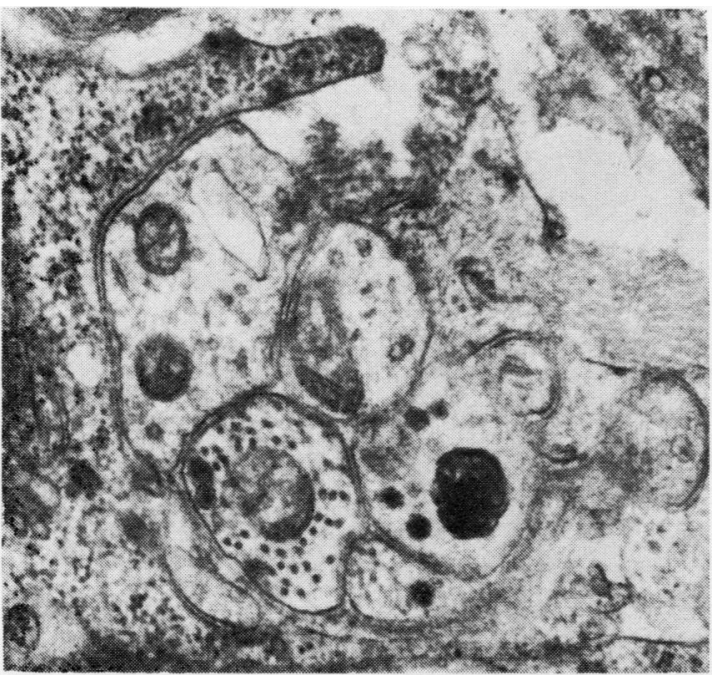

Fig. 9 Higher magnification of nerve process seen in Fig. $8 . \times 26600$.

junctions with the adjacent cuboidal cells. There is some interdigitation of the lateral plasma membranes and desmosome connections. The basal plasma membrane is discontinuous and clear, and dense core vesicles appear external to its cytoplasm. In the interstitium adjacent to this cell an undifferentiated mesenchymal cell, probably destined to become smooth muscle, lies between the $\mathrm{K}$-cell and the nerve fibre, which consists of several separate cytoplasmic processes, most of which contain mitochondria and microtubules. One of the processes contains three dense core vesicles of dimensions corresponding to the lower range of the dense core vesicles of the $\mathrm{K}$-cells. The whole bundle of processes rests within an arch of cytoplasm from an undifferentiated interstitial cell (Fig. 9).

$\mathrm{K}$-cells in mitosis are rarely seen. A possible post-mitotic figure is seen in Fig. 10a. The cell lies on the epithelial basement membrane of a bronchiole and contains the characteristic dense core vesicles, clear vesicles, ribosomes, mitochondria, and some rough endoplasmic reticulum in zonal arrangement. The clumped nuclear chromatin, and the cytoplasmic configuration of membranes sandwiching electron dense chromatin-like material, suggest an immediate post-mitotic stage. In other K-cells, features such as a primary cilium extending from a basal body, and stacks of remnant nuclear membranes within the cytoplasm (Fig. 10b), also suggest recent cell division.

\section{Discussion}

The observations presented here confirm those of previous studies that $\mathrm{K}$-type cells occur with relative frequency in the bronchi and bronchioles of developing human lungs (Rosan and Lauweryns, 1971; Hage, 1972a, b) and that they have welldifferentiated cytoplasm, which appears to be actively engaged in the synthesis of dense core vesicles.

The present report is the first to describe in detail the endocrine-like cells in the terminal bronchioles and saccules of human fetal lung. The significant observations are that the cells have extensive cytoplasmic processes within which the organelles seem to have a definite arrangement, as in similar cells more proximal in the bronchial tree. The cells appear to be engaged in active synthesis of the dense core vesicles and the variation in density of the cores may indicate either different stages in their development or even different secretory products (Lauweryns and Cokelaere, 1973; Cutz et al., 1974). The presence of dense core or clear vesicles outside the cell membrane, particularly in the intercellular spaces and in the adjacent interstitium, indicates that they are secreted. Moreover, the apparent gaps in the basal plasma membrane confirm this, or suggest some connection with interstitial elements.

Previous studies (Rosan and Lauweryns, 1971; Hage, 1972a, b; Cutz and Conen, 1972) on human fetal bronchiolar K-cells have established that they are neurosecretory cells belonging to the APUD system (Pearse, 1974). Lauweryns and Peuskens (1969) suggested that, in producing serotonin and other related amines and having a possible role in the kinin generating system, they might be responsible for circulatory adaptation at birth and in the newborn. Rosan and Lauweryns (1971), mentioning their presence in the intersaccular septa' of human fetal lung, suggested that they have a regulatory activity in the pulmonary microcirculation.

Although the content of the neurosecretory cells has not been determined from this study, their close association across the basement membrane with either smooth muscle cells of the terminal bronchioles or endothelial cells of the capillaries is suggestive of a functional association (Rosan and Lauweryns, 1971). In addition, the presence of secretory granules and clear vesicles in the intercellular spaces between epithelial cells supports the idea that they may have an influence on the neighbouring epithelial cells, but not that they are precursors of these, as suggested by Terzakis et al. (1972). Finally cytoplasmic contact with both 


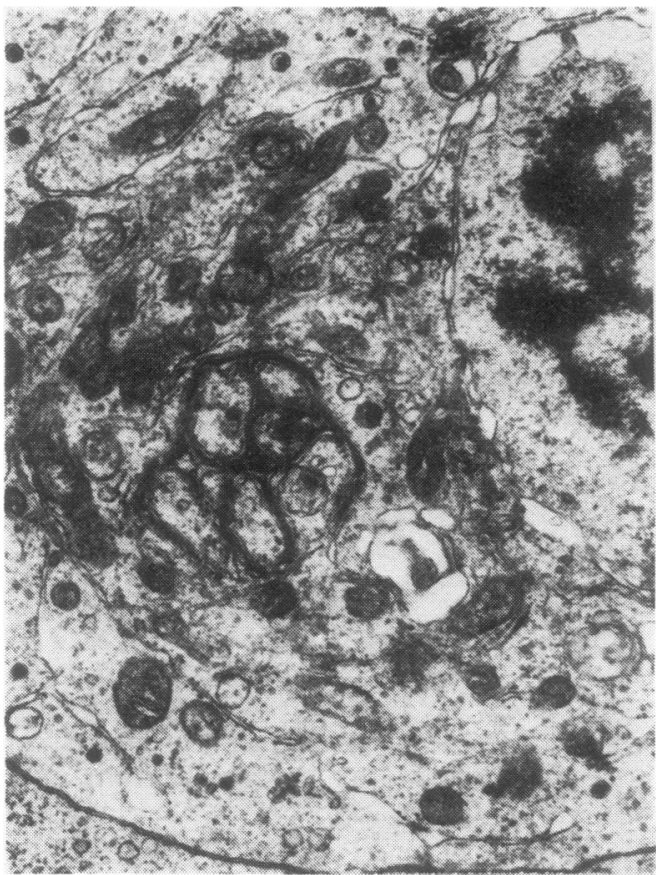

(a)

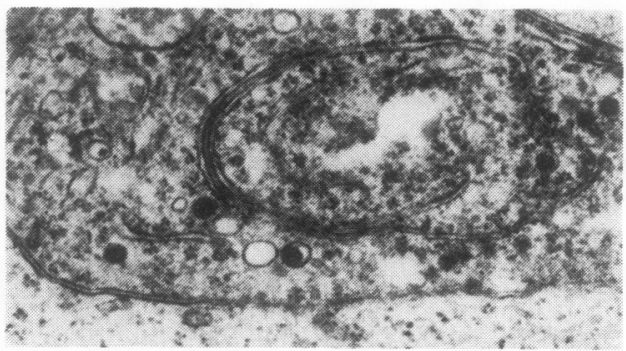

(b)

Fig. 10 (a) 22-week fetus. A possible post-mitotic $K$-cell can be identified by the numerous mitochondria and dense core vesicles. The nuclear chromatin is clumped, and within the cytoplasm is a configuration of membranes sandwiching electron dense chromatin-like material. $\times 15600$. (b) 22-week fetus. A stack of remnant nuclear membranes in a $K$-cell cytoplasmic process suggest recent cell division. $\times 20900$. 
intercellular spaces and the lumen suggests that the cells may also be sensitive to chemical changes in those areas.

From this study the groups of $\mathrm{K}$-cells in the bronchioles seem to be composed of neurosecretory cells similar to the single cells described. It is doubtful whether they correspond to the 'neuroepithelial bodies' described in human infants by Lauweryns and Peuskens (1972), in rabbits (Lauweryns et al., 1973), and in mice (Hung et al., 1973). In a study of neonatal rabbit and mice bronchioles, Lauweryns and Cokelaere (1973) found these organs to have both afferent and efferent innervation and their component cells to contain and secrete serotonin. They proposed that the cells provided 'an intrapulmonary hypoxiasensitive neuro(chemo-)receptor in addition to the central and peripheral (eg carotid body) chemoreceptors'. Moosavi and Heath (1973) found that bronchial Kulschitsky cells in neonatal rats discharged their granules in response to hypoxia. They also proposed either a chemoreceptor or endocrine function.

In the present study, the rare finding of a small nerve fibre in the wall of a terminal bronchiole (probably a prospective respiratory bronchiole) (Figs 8 and 9) raises the possibility that it is associated with a nearby $\mathrm{K}$-cell, thereby fulfilling a type of 'minireceptor' role in addition to the vasoactive one.

Finally, it is possible that $\mathrm{K}$-cells divide in the fetal lung. The evidence for this includes the clumping of chromatin in the nucleus, a cytoplasmic configuration of membranes sandwiching electron dense chromatin-like material (Haynes and Davies, 1973) and stacks of remnant nuclear membranes (McDougall, 1976).

I thank Professor J. F. Smith for his help in criticising the manuscript.

\section{References}

Campiche, M. A., Gautier, A., Hernandez, E. I., and Reymond, A. (1963). An electron microscope study of the development of human lung. Pediatrics, 32, 976-994.

Cutz, E., Chan, W., Wong, V., and Conen, P. E. (1974). Endocrine cells in rat fetal lungs. Ultrastructural and histochemical Study. Laboratory Investigation, 30, 458-464.

Cutz, E., and Conen, P. E. (1972). Endocrine-like cells in human fetal lungs: an electron microscopic study. Anatomical Record, 173, 115-122.

Hage, Esther (1972a). Electron microscopic identification of endocrine cells in the bronchial epithelium of human fetuses. Acta Pathologica Microbiologica Scandinavica, Section (A), 80, 143-144.
Hage, Esther (1972b). Endocrine cells in the bronchial mucosa of human fetuses. Acta Pathologica Microbiologica Scandinavica, Section (A), 80, 225-234.

Haynes, M. E., and Davies, H. G. (1973). Observations on the origin and significance of the nuclear envelope-limited monolayers of chromatin unit threads associated with the cell nucleus. Journal of Cell Science, 13, 139-171.

Hung, K. S., Hertweck, M. S., Hardy, J. D., and Loosli, C. G. (1973). The ultrastructure of nerve and associated cells in bronchiolar epithelium of mouse lung. Journal of Ultrastructure Research, 43, 426-437.

Jeffery, P. K.. and Reid, Lynne (1975). New observations of rat airway epithelium: a quantitative and electron microscopic study. Journal of Anatomy, 120, 295-320.

Lauweryns, J. M. (1970). Hyaline membrane disease in newborn infants. Macroscopic, radiographic and light and electron microscopic studies. Human Pathology, 1, 175-204.

Lauweryns, J. M., and Cokelaere, M. (1973). Hypoxiasensitive neuro-epithelial bodies. Intrapulmonary secretory neuroreceptors modulated by the C.N.S. Zeitschrift für Zellforschung und mikroskopische anatomie, 145, 521-540.

Lauweryns, J. M., Cokelaere, M., and Theunynck, P. (1973). Serotonin producing neuroepithelial bodies in rabbit respiratory mucosa. Science, 180, 410-413.

Lauweryns, J. M., and Peuskens, J. C. (1969). Argyrophil (kinin and amine producing?) cells in human infant airway epithelium. Life Science, 8, 577-585.

Lauweryns, J. M., and Peuskens. J. C. (1972). Neuroepithelial bodies (neuroreceptor or secretory organs?) in human infant bronchial and bronchiolar epithelium. Anatomical Record, 172, 471-482.

McDougall, Jennifer (1976). Some ultrastructural aspects of mitosis and early differentiation in human fetal lung. Journal of Cell Science, 22, 67-74.

McDougall, Jennifer, and Smith, J. F. (1975). The development of the human type 2 pneumocyte. Journal of Pathology, 115, 245-251.

Moosavi, H., Smith, P., and Heath, D. (1973). The Feyrter cell in hypoxia. Thorax, 28, 729-741.

Pearse, A. G. E. (1974). The APUD cell concept and its implications in pathology. Pathology annual, 9, 27-41.

Rosan, R. C., and Lauweryns, J. M. (1971). Secretory cells in the premature human lung lobule. Nature, 232, 60-61.

Rosan, R. C., and Lauweryns, J. M. (1972). Mucosal cells of the small bronchioles of prematurely born human infants (600-1700 g.). Beitrage zur Pathologie, 147, 145-174.

Terzakis, J. A., Sommers, S. C., and Andersson, B. (1972). Neurosecretory appearing cells of human segmental bronchi. Laboratory Investigation, 26, $127-132$.

Requests for reprints to: Dr. Jennifer McDougall, Department of Morbid Anatomy, University College Hospital Medical School, London WC1E 6JJ. 